\title{
Valproic acid use in pediatric partial epilepsy after initial medication failure
}

\author{
Russell P. Saneto ${ }^{1,2}$, Prakash Kotagal ${ }^{2,3}$, A. David Rothner $^{3}$, \\ Jill Baker $^{2}$, Laura L. Kotagal ${ }^{3}$ \\ ${ }^{1}$ Division of Pediatric Neurology, Children's Hospital and Regional \\ Medical Center and The University of Washington, U.S.A. \\ Department of Neurology, Sections of ${ }^{2}$ Pediatric Epilepsy and ${ }^{3}$ Pediatric \\ Neurology Cleveland Clinic Foundation, Euclid Avenue, Cleveland, Ohio U.S.A.
}

\begin{abstract}
We retrospectively identified 46 children and adolescents with partial epilepsy, who failed prior treatment with phenytoin, carbamazepine, phenobarbital and/or primidone. All patients were subsequently treated with valproate (VPA). Outcome measurements were stratified into seizure type, simple partial seizures (SPS), complex partial seizures (CPS) or secondarily generalized seizures (SGS), or lesions detected by magnetic resonance images (MRI). The majority of the patients had a $\geq 75 \%$ reduction of seizure frequency. Complete seizure freedom was achieved in $7(15 \%)$ patients. "Worthwhile" seizure reduction, defined as $\geq 75 \%$ decrease (but $<100 \%$ ) in seizure activity, was found in 30 (65\%) patients. VPA was efficacious in SPS (2/2), CPS $(9 / 12)$ and SGS (26/32) patients. Patients with MRI lesions of vascular insult (6/6), mesial temporal lobe sclerosis $(8 / 10)$, or malformation of cortical development (6/7) had a higher response percentage than those patients with normal MRI findings (11/15) and tumors (5/7). Although the numbers of patients used in this study were too small for statistical significance, our results suggest that VPA is a useful medication for previous medication failures in childhood
\end{abstract}

Correspondence: Prakash Kotagal, M.D.,

Department of Neurology,

Section of Pediatric Epilepsy,

Cleveland Clinic Foundation,

9500 Euclid Avenue, Cleveland,

Ohio 44195 U.S.A.

Fax: (216) 445-6617.

E-mail: kotagap@ccf.org

Received: July 04, 2004.

Revised: September 14, 2004

Accepted: September 15, 2004. partial seizures across seizure types as well as MRI lesions. There is some suggestion that a higher percentage of patients with MRI lesions may respond favorably to VPA than patients with non-lesional partial seizures and tumors. ( $\mathrm{J}$ Pediatr Neurol 2004; 2(4): 199-203).

Key words: partial seizures, valproic acid, children, magnetic resonance imaging, anti-epileptic medication.

\section{Introduction}

Valproate (VPA) is effective in the treatment of generalized-onset seizures, especially in children $(1,2)$. Many consider VPA to be the medication of choice for generalized epilepsies of childhood and adolescence (3). However, the usefulness of VPA, either as monotherapy or combined therapy is not well defined in the treatment of simple partial seizures (SPS), complex partial seizures (CPS) alone or with secondarily generalized tonicclonic seizures (SGS). There is little information concerning response to VPA in symptomatic or cryptogenic partial epilepsy, defined by magnetic resonance imaging (MRI) and partial seizures.

There are conflicting reports on the usefulness of VPA in partial epilepsy. There have been several studies investigating drug therapy efficacy in treating newly diagnosed partial epilepsy. The Veterans Administration Cooperative Study showed that carbamazepine or phenytoin monotherapy has the best overall combination of efficacy and freedom from adverse effects (4). However, other studies have reported VPA to be equally effective in the treatment of adult partial epilepsies (5-8). In a few small studies, VPA was found to have efficacy similar to phenytoin or carbamazepine $(9,10)$.

VPA has been shown to be effective after previous therapeutic failure in partial epilepsies. 
Several small studies looked at mixed populations of adults and children and found VPA to be efficacious in seizure reduction (11-14). One large study of 410 children and adults comparing phenobarbital, phenytoin, carbamazepine, and VPA showed no significant differences in the proportions of patients achieving a 1-year remission $(15,16)$. In one pediatric study, Coulter et al. (17) found VPA to be effective in both generalized $(n=96)$ and "focal" seizures $(n=23)$ after previous medication failure. Sherard et al. (18) demonstrated that VPA, as addon therapy, reduced seizure frequency by $\geq 75 \%$ in $22 / 38$ pediatric patients with partial epilepsy.

To date, the effectiveness of VPA in children and adolescents within the various partial epileptic seizure classifications is unclear, especially after previous therapeutic failure. Furthermore, the response to VPA in the various etiologies of partial epilepsy is unknown. We investigated our database for those patients identified to have SPS, CPS, or SGS on the basis of EEG, seizure semiology, and magnetic resonance images (MRI). All patients were judged to be intractable, since their seizures were not controlled despite adequate treatment with phenytoin, carbamazepine, primidone or phenobarbital. We describe their response to VPA with respect to various types of partial seizures and MRI defined abnormalities.

\section{Materials and Methods}

\section{Study criteria}

We based our inclusion criteria by patient history, EEG and MRI lesion. Each patient had at least one EEG and MRI at our institution, unless otherwise stated. All patients had partial seizures as defined by the International League Against Epilepsy (ILAE) seizures classification (19). Although each patient had partial seizures, they varied considerably in seizure semiology, epilepsy etiology, and/or syndrome. Seizure frequency was based on parental observation recorded during follow-up office visits, recorded telephone contact, and hospital admission history.

\section{Patients}

We retrospectively analyzed the medical charts of 46 patients who had been treated with VPA and had partial epilepsy. There were 23 males and 23 females with ages ranging from 18 months to 20 years of age. All but one patient was 18 years or younger, with $>50 \%(n=24)$ being age 12 years or less. All patients had failed medical management on monotherapy with phenytoin, carbamazapine, or phenobarbital prior to VPA therapy. Nine patients failed initial monotherapy with phenytoin, and 17 failed carbamazepine. Seventeen of the 26 patients, who failed monotherapy, also failed the subsequent use of either phenytoin or carbamazepine when switched to the new medication. Five patients had a history of phenobarbital and/or primidone use as initial therapy. A total of 20 patients of these 31 patients had been on phenobarbital and/or primidone at one time or another. In all, 31 of the 46 patients had been on various combinations of anti-epileptic drugs prior to the addition of VPA. By chart review, all patients on mono- and combination therapy had levels within published therapeutic levels. All patients, while on VPA, had therapeutic levels of VPA. Patients were classified by seizure type based on the ILAE seizure classification (19): there were 2 patients with SPS, 12 patients with CPS, and 32 patients with SGS. We excluded patients who had benign focal epilepsy of childhood.

\section{Magnetic resonance imaging}

All but one patient (see below) had brain MRI scans. A 1.5 Tesla MRI scanner was used to obtain all images. Sequences included a sagittal T1-weighted image scout film, followed by double-echo T2weighted images (TR 2500/TE 25, 90/25-cm field of view/192 X 256 matrix/5-mm slice thickness/2-mm gap) performed in the coronal, axial, and sagittal planes. A neuroradiologist evaluated all MRI images.

\section{Partial epilepsies}

There were 31 patients with symptomatic partial epilepsy. Seven patients with low-grade tumors, seven with malformation of cortical development (MCD), six who had arterio-venous malformations (AVM) or cerebral infarcts, one had bacterial encephalitis, and nine with mesial temporal sclerosis. One patient had previously diagnosed Rasmussen's encephalitis and did not have a MRI at our institution. The initial MRI scan of the brain in the Rasmussen's patient was reported as normal. There were 15 patients with cryptogenic partial epilepsy who had no neuroimaging abnormality detected.

\section{Seizure outcome measurements}

The medical charts were analyzed to determine seizure outcome. Seizure outcome was divided into three groups: seizure-free, "worthwhile " seizure reduction $(\geq 75 \%$ seizure reduction but less than $100 \%$ ), and those with $<75 \%$ seizure reduction or not improved (modified from 20). Estimation of response was based on seizure frequency over a minimum of 30 days, and for comparison, frequency was averaged over 30-day periods. Patients whose response to VPA lasted less than 30 days were also regarded as not improved. Patients who responded to VPA had seizure improvement that persisted more than 90 days. Patients who had not been on VPA therapy for at least 30 days were excluded 
Table 1. Seizure-free outcome in patients with distinct MRI lesions within various ILAE classifications of partial seizures

\begin{tabular}{|c|c|c|c|c|c|c|}
\hline $\begin{array}{l}\text { Seizure } \\
\text { types }\end{array}$ & $\begin{array}{l}\text { Malformation } \\
\text { of cortical } \\
\text { development }\end{array}$ & Vascular & $\begin{array}{l}\text { Mesial } \\
\text { temporal } \\
\text { sclerosis }\end{array}$ & Tumor & $\begin{array}{l}\text { Normal } \\
\text { MRI }\end{array}$ & Patients \\
\hline Simple partial seizures & 1 & - & - & - & - & $1 / 2$ \\
\hline Complex partial seizures & - & 1 & - & - & 1 & $2 / 12$ \\
\hline \multirow{2}{*}{$\begin{array}{l}\text { Secondarily generalized } \\
\text { seizures }\end{array}$} & - & 1 & 1 & 1 & 1 & $4 / 32$ \\
\hline & $1 / 7$ & $2 / 6$ & $1 / 10$ & $1 / 7$ & $2 / 15$ & $7 / 46^{*}$ \\
\hline
\end{tabular}

* One patient had bacterial meningitis and did not become seizure-free on VPA.

from the study. Our determination of "worthwhile" seizure reduction is based on seizure frequency and not quality of life issues. This aspect of seizure control needs to be addressed in the future.

\section{Results}

\section{Outcome}

Outcome measurements were categorized into a modification of Engel's Classification (see above for description). Overall, 37 patients responded to VPA with seizure freedom or worthwhile response. Seven patients became seizure-free. There was $\geq$ $75 \%$ seizure control, but not seizure freedom, in 30 patients while 9 patients had little or no response.

The duration of seizure freedom ranged from 0.5 to 10 years with a median of 3 years. In the group of 7 patients who became seizure-free, 2 are currently on no medication and 5 remain on VPA monotherapy. Of 30 patients who had a worthwhile reduction in seizure activity, four are currently on VPA monotherapy, while the remaining 26 are on combination therapy regimen that includes VPA. The duration of worthwhile response was from 0.3 to 12 years with a median of 2 years. There were 7 patients that had less than a $75 \%$ reduction in seizure frequency to VPA treatment but who are still taking VPA as part of their medical management, while 2 patients are on other medication(s) not including VPA. Both these patient groups were considered non-responders.

\section{ILAE seizure classification and outcome}

There were little differences in the response to VPA among different ILAE classifications (Tables 1 and 2). The 2 patients having SPS responded to VPA, by either becoming seizure-free $(n=1)$ or having $\geq 75 \%$ seizure control $(n=1)$. In patients with CPS, a large percentage $(75 \%)$ responded with either seizure freedom or $\geq 75 \%$ seizure control. Of the 12 CPS patients, 2 patients became seizure-free, while 7 patients had $\geq 75 \%$ seizure control. Three patients in this classification did not respond favorably to
VPA. There were 32 patients who had SGS. Four of these patients became seizure-free with 22 patients having a worthwhile reduction in seizure frequency. The remaining 6 patients had no response to VPA either as monotherapy or in combination therapy.

\section{MRI lesion and outcome}

Further subdividing patients by MRI lesions, MCD, tumor, AVM/infarction, infection (normal MRI scan), mesial temporal sclerosis, and no abnormality, revealed more responsive and less responsive subgroups (Tables 1 and 2). There were 30 patients with definable lesions on MRI scanning. Of these patients, $24(80 \%)$ patients had reductions in seizure activity. Five patients became seizurefree and 19 patients had $\geq 75 \%$ seizure control. Fifteen patients had normal or no definable lesions on MRI scanning. Only $11(73 \%)$ patients in this group responded with decreased seizure activity, 2 patients became seizure-free and 9 patients had $\geq$ $75 \%$ seizure control.

All six of the patients with AVM/infarction lesions responded favorably to VPA. Two patients became seizure-free, while four others had $\geq 75 \%$ seizure control. In the subgroup of patients with MCD, six of seven patients had a worthwhile reduction in seizure activity. The one non-responder in this latter group had hemimegencephaly. One patient became seizure-free and 5 patients had a worthwhile reduction in seizure frequency. Eight of the 10 patients with mesial temporal sclerosis had $\mathrm{a} \geq 75 \%$ reduction in seizure activity. There was 1 patient who became seizure-free, while 7 had a worthwhile reduction in their seizure frequency. Interestingly, 7 of these patients went on to have surgery in an attempt to become completely seizurefree. Of the 7 patients who elected surgery, 6 are currently seizure-free or only having infrequent auras, while the other patient has a worthwhile decrease in seizure frequency (data not shown).

There were seven patients who had a low-grade tumor. One of these patients became seizure-free, while 4 patients had a worthwhile reduction in 
Table 2. Worthwhile response in patients with distinct MRI lesions within various ILAE classifications of partial seizures

\begin{tabular}{|c|c|c|c|c|c|c|}
\hline $\begin{array}{l}\text { Seizure } \\
\text { types }\end{array}$ & $\begin{array}{l}\text { Malformation } \\
\text { of cortical } \\
\text { development }\end{array}$ & Vascular & $\begin{array}{l}\text { Mesial } \\
\text { temporal } \\
\text { sclerosis }\end{array}$ & Tumor & $\begin{array}{l}\text { Normal } \\
\text { MRI }\end{array}$ & Patients \\
\hline Simple partial seizures & - & - & 1 & - & - & $1 / 2$ \\
\hline Complex partial seizures & 2 & - & 2 & - & 3 & $7 / 12$ \\
\hline \multirow{2}{*}{$\begin{array}{l}\text { Secondarily generalized } \\
\text { seizures }\end{array}$} & 3 & 4 & 5 & 3 & 6 & $22^{+} / 32$ \\
\hline & $5 / 7$ & $4 / 6$ & $7 / 10$ & $4 / 7$ & $9 / 15$ & $30 * / 46 *$ \\
\hline
\end{tabular}

* One patient had bacterial meningitis, SGS, and had a worthwhile response to VPA.

${ }^{+}$Another patient had Rausmussen encephalitis, whose MRI scan was initially normal and the major seizure semiology was SGS.

their seizure activity. Of the 2 patients who did not respond, 1 had ganglioglioma and the other a dysembryoplastic neuroepithelial tumor. Only one patient had a history of infection, unspecified bacterial encephalitis, in this study. This patient had a worthwhile reduction of seizure activity and remains on VPA.

\section{Surgery}

There were 16 patients who elected to undergo epilepsy surgery to become seizure-free. Seven patients had the seizure semiology, EEG, and MRI findings of mesial temporal lobe epilepsy. In the remaining group of patients, there were five patients with MCD (including the patient with hemimegencephaly), two patients with a tumor, one patient with an AVM/infarction, and one patient with Rasmussen's encephalitis.

\section{Untoward effects}

Thirteen of the patients commented on the side effects of VPA. In order of frequency, five patients complained of weight gain, four patients of lethargy, two patients of tremor, two patients of hair loss, and one patient had gastrointestinal upset. Two patients had multiple complaints; one patient had low platelet counts and weight gain, while the second patient had hair loss and elevated liver enzyme values.

\section{Discussion}

Studies have demonstrated that VPA is comparable to either phenytoin or carbamazepine in the control of partial epilepsy, when initiated in the untreated or under-treated pediatric patient $(9,10,21)$. Several studies that included both children and adult patients have shown that VPA can be efficacious in seizure control of partial epilepsy, after failure with other medications $(8,11,13-16)$. In each of these latter studies, the number of children and adolescents is unknown, with the exception of the study by Richens and Ahmad (11) in which there was only a single adolescent in the study. Several smaller pediatric studies, the series of Coulter et al. (17) $(n=23)$ and Sherard et al. (18) $(n=38)$, have shown that VPA can be an effective treatment after previous medication failure in partial epilepsy. However, VPA efficacy in the treatment of specific types of partial seizures, or partial symptomatic epilepsy due to specific MRI lesions in the children with intractable partial seizures, remains unknown. We have corroborated other studies to show that in children and adolescents who failed previous monotherapy or combination therapy, VPA is a useful medication for the control of seizures due to partial epilepsy. To our knowledge, our study is the largest to look at children and adolescents with various types of partial seizures. Over $80 \%$ of the patients had $\geq$ $75 \%$ seizure control. In similar but smaller pediatric patient populations, Sherard et al. (18) showed a 55\% response rate and Coulter et al. (17) showed a 57\% response rate of $\geq 75 \%$ or better seizure reduction using VPA. These latter studies classified partial seizures as "focal," "psychomotor," or as "partial epilepsy." Studies by Dean and Penry (13) indicated that VPA was efficacious in patients of mixed ages with SGS. Our study suggested less response rates with SGS types. Overall, there is little difference in response to the various ILAE classifications of epileptic seizures. The broad classification criteria may dilute the ability to stratify various types of partial seizures into VPA responsive categories. A more precise classification scheme might allow better correlation with seizure medication efficacy and seizure type. It is not entirely clear why our study showed a more robust response to VPA than the other studies. Confounding factors such as sample size, selection bias, and patient population may influence the outcome.

There was a differential response to VPA to defined MRI lesions, suggesting the possibility 
that seizure etiology might be important in selecting an efficacious medication. Patients with a definitive lesion detected by MRI, symptomatic partial epilepsy, had a more robust response to VPA than cryptogenic patients with a normal MRI. Responsiveness to VPA also tended to segregate into MRI lesion categories. Patients with MCD, $\mathrm{AVM}$ /infarction, and mesial temporal lobe sclerosis responded better compared to those with a tumor. We found that patients with mesial temporal sclerosis responded better to VPA than previously described by Richens and Ahmad (11). There was at least an $87 \%$ response rate in the non-neoplastic group, compared to a $71 \%$ response rate in the tumor group. Within particular tumor types, there was also a differential response. All of the tumors were non-aggressive; however, only the slow growing gliomas responded to treatment. Patients with a ganglioglioma or dysembryoplastic neuroepithelial tumor did not respond to VPA. Whether responses differ depending on tumor type, size, grade, or location can only be determined in much larger studies.

Although patient numbers are small, our study suggests that children with various etiologies of partial epilepsy might respond to VPA differentially. Insufficient numbers of patients preclude our making firm statements. However, the trends suggest that treatment efficacy may be related to seizure etiology, but not ILAE classifications of partial seizures. A better classification of epileptic syndromes on the basis of seizure semiology, neuroimaging findings, and genetics may allow us to better judge the differential response of patients to various anti-epileptic drugs.

\section{Acknowledgements}

This project was supported in part by Abbott Laboratories.

\section{References}

1. Jeavons PM, Clark JE. Sodium valproate in the treatment of epilepsy. Br Med J 1974; 2: 584-586.

2. Chadwick DW. Valproate monotherapy in the management of generalized and partial seizures. Epilepsia 1987; 28: S12-S17.

3. Jeavons PM, Clark JE, Maheshwari MC. Treatment of generalized epilepsies of childhood and adolescence with sodium valproate ("epilim"). Dev Med Child Neurol 1977; 19: 9-25.

4. Mattson RH, Cramer JA, Collins JF. A comparison of valproate with carbamazepine for the treatment of complex partial seizures and secondarily generalized tonic-clonic seizures in adults. The Department of Veterans Affairs Epilepsy Cooperative Study No. 264 Group. N Engl J Med 1992; 327: 765-771.

5. Callaghan N, Kenny RA, O'Neill B, Crowley M, Goggin T. A prospective study between carbamazepine, phenytoin and sodium valproate as monotherapy in previously untreated and recently diagnosed patients with epilepsy. J Neurol Neurosurg Psychiatry 1985; 48: 639-644.

6. Turnbull DM, Howel D, Rawlins MD, Chadwick DW Which drug for the adult epileptic patient: phenytoin or valproate? Br Med J 1985; 290: 815-819.

7. Heller AJ, Chesterman P, Elwes RDC, et al. Phenobarbitone, phenytoin, carbamazepine, or sodium valproate for newly diagnosed adult epilepsy: a randomised comparative monotherapy trial. J Neurol Neurosurg Psychiatry 1995; 58: 4450 .

8. Beydoun A, Sackellares JC, Shu V. Safety and efficacy of divalproex sodium monotherapy in partial epilepsy: a double-blind, concentration-response design clinical trial. Depakote Monotherapy for Partial Seizures Study Group. Neurology 1997; 48: 182-188.

9. Verity CM, Hosking G, Easter DJ. A multicentre comparative trial of sodium valproate and carbamazepine in paediatric epilepsy. The Paediatric EPITEG Collaborative Group. Dev Med Child Neurol 1995; 37: 97-108.

10. deSilva M, MacArdle B, McGowan M, et al. Randomised comparative monotherapy trial of phenobarbitone, phenytoin, carbamazepine, or sodium valoproate for newly diagnosed childhood epilepsy. Lancet 1996; 347: 709-713.

11. Richens A, Ahmad S. Controlled trial of sodium valproate in severe epilepsy. Br Med J 1975; 4: 255 256.

12. Crawford P, Chadwick D. A comparative study of progabide, valproate, and placebo as add-on therapy in patients with refractory epilepsy. J Neurol Neurosurg Psychiatry 1986; 49: 1251-1257.

13. Dean JC, Penry JK. Valproate monotherapy in 30 patients with partial seizures. Epilepsia 1988; 29: 140-144.

14. Willmore LJ, Shu V, Wallin B. Efficacy and safety of add-on divalproex sodium in the treatment of complex partial seizures. The M88-194 Study Group. Neurology 1996; 46: 49-53.

15. Reynolds EH, Heller AJ, Chadwick D. Valproate versus carbamazepine for seizures. N Engl J Med 1993; 328: 207-208

16. Reynolds EH. Comparative monotherapy trials of four major antiepileptic drugs in adults and children (MRC trial). In: Brodie MJ (ed). Valproate: New Milestones. London: Franklin Scientific Publications, 1992, pp 16-19.

17. Coulter DL, Wu H, Allen RJ. Valproic acid therapy in childhood epilepsy. JAMA 1980; 244: 785-788.

18. Sherard ES Jr, Steiman GS, Couri D. Treatment of childhood epilepsy with valproic acid: results of the first 100 patients in a 6-month trial. Neurology 1980; 30: 31-35.

19. Proposal for classification of epilepsies and epileptic syndromes. Commission on Classification and Terminology of the International League Against Epilepsy. Epilepsia 1985; 26: 268-278.

20. Engel J. Outcome with respect to epileptic seizures. In: Engel J (ed). Surgical treatment of the epilepsies. New York: Raven Press, 1987, pp 553-571.

21. Coppola G. Treatment of partial seizures in childhood: an overview. CNS Drugs 2004; 18: 133156. 